\section{Impact of preexisting dengue immunity on Zika virus emergence in a dengue endemic region}

\author{
Isabel Rodriguez-Barraquer ${ }^{1 *}$, Federico Costa ${ }^{2,3,4 *}$, Eduardo J. M. Nascimento ${ }^{5 *}$, \\ Nivison Nery Júnior ${ }^{2,3}$, Priscila M. S. Castanha ${ }^{6,7}$, Gielson Almeida Sacramento ${ }^{3}$, \\ Jaqueline Cruz $^{3}$, Mayara Carvalho ${ }^{3}$, Daiana De Olivera ${ }^{3}$, José E. Hagan ${ }^{3,4}$, \\ Haritha Adhikarla ${ }^{4}$, Elsio A. Wunder Jr. ${ }^{3,4}$, Danilo F. Coêlho ${ }^{6,8}$, Sasha R. Azar ${ }^{9}$, \\ Shannan L. Rossi $^{10}$, Nikos Vasilakis ${ }^{11}$, Scott C. Weaver ${ }^{10}$, Guilherme S. Ribeiro ${ }^{2,3,12}$, \\ Angel Balmaseda ${ }^{13,14}$, Eva Harris ${ }^{15}$, Maurício L. Nogueira ${ }^{16}$, Mitermayer G. Reis ${ }^{3,4,12}$, \\ Ernesto T. A. Marques ${ }^{5,6,17 *}$, Derek A. T. Cummings ${ }^{18,19 *}+$, Albert I. Ko ${ }^{3,4 *} \dagger$
}

The clinical outcomes associated with Zika virus (ZIKV) in the Americas have been well documented, but other aspects of the pandemic, such as attack rates and risk factors, are poorly understood. We prospectively followed a cohort of 1453 urban residents in Salvador, Brazil, and, using an assay that measured immunoglobulin G3 (IgG3) responses against ZIKV NS1 antigen, we estimated that $73 \%$ of individuals were infected during the 2015 outbreak. Attack rates were spatially heterogeneous, varying by a factor of 3 within a community spanning 0.17 square kilometers. Preexisting high antibody titers to dengue virus were associated with reduced risk of ZIKV infection and symptoms. The landscape of ZIKV immunity that now exists may affect the risk for future transmission.

I here are many uncertainties about the dynamics of Zika virus (ZIKV) outbreaks in large, immunologically naïve populations. There is also continued uncertainty surrounding the circumstances that allowed ZIKV to emerge in American populations and the future of ZIKV transmission in the Americas and elsewhere (1). The association between ZIKV infection and microcephaly cases makes it important to determine whether future cases can be expected or whether immunity levels in a population are likely to prevent major future transmission. These uncertainties underscore the need to characterize infection rates of the 2015-2016 epidemic $(1,2)$.

One key unanswered question is the role of preexisting immunity to dengue virus (DENV), an endemic pathogen that is genetically and antigenically similar to ZIKV and has been hypothesized to both promote (3) and suppress ZIKV emergence (4). Numerous in vitro studies have shown enhanced ZIKV infection in the presence of DENV antibodies, but results from in vivo studies have been less clear $(3,5-8)$. Immunity to DENV has been shown experimentally to neutralize and protect against ZIKV infection (9-11) and may therefore reduce the probability of successful establishment of ZIKV in a DENVimmune population (12). Yet to date, the influence of preexisting DENV immunity on susceptibility or resistance to ZIKV infection has not been rigorously evaluated in human populations.

Ideally, to characterize the interactions between ZIKV and DENV requires prospectively monitoring individuals of different DENV serostatus and measuring any ZIKV infection. However, a large proportion of ZIKV and DENV infections are asymptomatic, and symptomatic infections have a nonspecific clinical presentation, making diagnosis challenging. Therefore, infection rates in populations can only be accurately quantified using serological measures. At present, there is a lack of commercially available serological tests that can measure antibody responses to DENV and ZIKV with sufficient sensitivity and specificity because of cross-reactivity between the two related viruses (13-16). These limitations have hampered efforts to quantify the extent of the American epidemic and evaluate interactions between DENV and ZIKV.

\section{High ZIKV attack rates in an urban population}

We had the opportunity to prospectively characterize the dynamics of ZIKV transmission in Pau da Lima, a community in the city of Salvador, where we have been conducting long-term cohort investigations of urban slum health problems (17-19). Northeast Brazil has been known to be hyperendemic for DENV for more than 30 years. It was also the epicenter of the 2015 Zika epidemic (20-22). Multiple rounds of sample collection occurred in our cohort since 2013, before and after the majority of clinical Zika cases were detected in this community and the city (23). Here, we quantified the attack rate of ZIKV during the 2015 outbreak using data from 1453 individuals in this cohort (Fig. 1 and tables S1 and S2). In a subset of 642 individuals for whom dengue immunity data were available (table S1), we investigated the impact of prior DENV immunity on the risk of ZIKV. Figure 2 shows the timing of sample collection before and after the period of the Zika outbreak.

We identified ZIKV infections with an assay that measures immunoglobulin G3 (IgG3) responses to the ZIKV NS1 protein (anti-ZIKV NS1 IgG3). The sensitivity and specificity of the assay were estimated to be $85 \%$ and $97 \%$, respectively, by testing a random subset of samples using plaque-reduction neutralization tests (PRNTs) (Fig. 2). We performed additional validation of this assay to ensure that cross-reactivity did not bias our inferences, using samples from before and after ZIKV emergence in the study population (Fig. 2) as well as external validation using patient samples from polymerase chain reactionconfirmed infections from multiple locations (figs. S2 and S3 and table S3).

Samples collected in October 2014 and March 2015, prior to the epidemic in Salvador (fig. S1), were mostly seronegative by the anti-ZIKV NS1 IgG3 assay [October 2014: 7\% seropositive; $95 \%$ confidence interval (CI), 4 to 10\%; $n=249$; March 2015: $8 \% ; 95 \%$ CI, 6 to $10 \% ; n=675$ ], as well as by PRNT [ $1 \%$ seropositive ( $95 \%$ CI, 0 to $4 \%$ ), $n=101$ ], suggesting little or no transmission before this date. In contrast, $63 \%$ (95\% CI, 60 to $65 \% ; n=1453$ ) of samples collected in October 2015, 5 months after recognized clinical cases peaked, were positive, indicating infection of the majority of individuals in the cohort (Fig. 3A). Adjusting for the sensitivity and specificity of the IgG3 assay

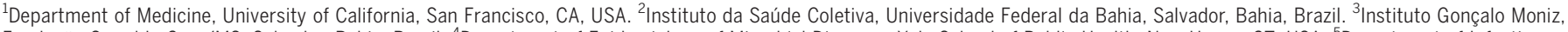

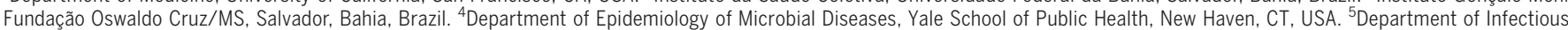

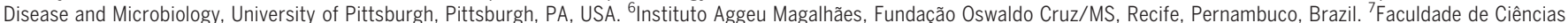

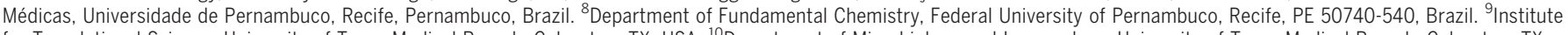



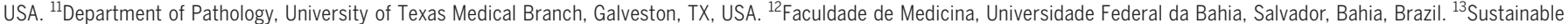

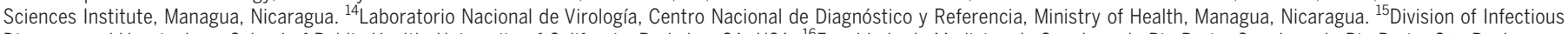
Diseases and Vaccinology, School of Public Health, University of California, Berkeley, CA, USA. ${ }^{16}$ Faculdade de Medicina de São Jose do Rio Preto, São Jose do Rio Preto, São Paulo, Brazil. ${ }^{17}$ Graduate School of Public Health, University of Pittsburgh, Pittsburgh, PA, USA. ${ }^{18}$ Department of Biology, University of Florida, Gainesville, FL, USA. ${ }^{19}$ Emerging Pathogens Institute, University of Florida, Gainesville, FL, USA.

*These authors contributed equally to this work.

†Corresponding author. Email: albert.ko@yale.edu (A.I.K.); datc@ufl.edu (D.A.T.C.)
} 

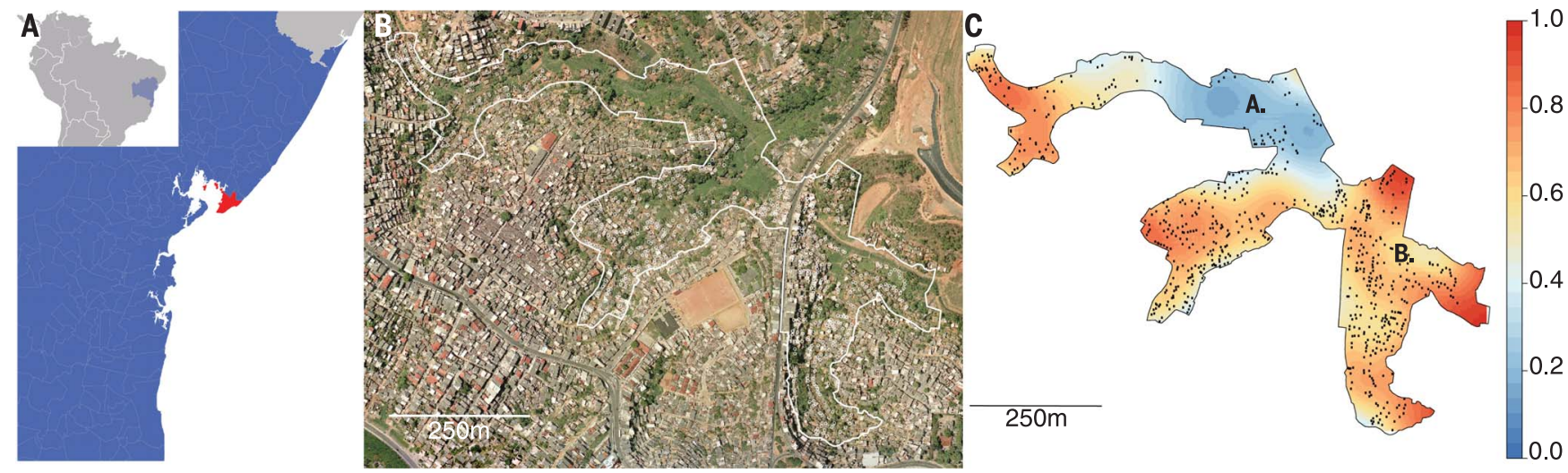

Fig. 1. Location of the study. (A) Location of the city of Salvador within the state of Bahía and within Brazil. (B) Satellite image of the Pau da Lima community study site in Salvador. (C) Spatial heterogeneity in the proportion of the samples that were found to be ZIKV IgG3-positive (indicated by colors) in October 2015. The smoothed surface was generated using a generalized additive model that included latitude, longitude, and altitude as predictors. Study areas A and B are indicated. Black dots denote locations of study households.
(Fig. 2C and table $\mathrm{S} 3$ ), this level of seropositivity corresponds to an attack rate of $73 \%$ (95\% CI, 70 to $76 \%$ ) and is consistent with the high seropositivity rate found in nonrandom subsets of individuals previously tested in Salvador (24).

\section{Heterogeneity in attack rates at fine spatial scales}

Despite the high seropositivity rates across all age groups (Fig. 3A), attack rates were not spatially uniform across the population that resided within the $0.17-\mathrm{km}^{2}$ study site (Fig. 1C). The attack rate among individuals living in the valley located in the northeast sector of the study area (area A, Fig. 1C) was $29 \%$ (95\% CI, 19 to $41 \% ; n=85)$, as compared to $83 \%$ (95\% CI, 76 to $90 \% ; n=241$ ) among individuals living in the southeast corner (area B, Fig. 1C). Similar spatial heterogeneity in DENV transmission has been reported within this community and elsewhere $(21,25)$. This variation could be due to differences in populations of mosquito vectors or in exposure of people living under different conditions.

On the basis of attack rates, we estimated that the basic reproductive number $\left(R_{0}\right)$ of ZIKV in this setting was 1.8 (95\% CI, 1.7 to 1.9 ) but ranged between 1.2 and 2.1 between areas of the study site. This result is consistent with estimates obtained using case reports from the early epidemic, which suggest that reproductive numbers fell from 2.2 to 0.7 from April 2015 to July 2015 (average of 2.0; 95\% CI, 1.9 to 2.1) (fig. S1) (23). These results are also within the range of previously published estimates for ZIKV $(2,24,26-28)$ as well as the basic reproductive number for DENV (2.3; 95\% CI, 2.1 to 2.4), which was estimated using age-stratified serological data collected prior to the ZIKV outbreak (Fig. 3B and supplementary materials). The reduction in reproductive numbers and the large reduction in case numbers reported in the city of Salvador in 2016 and 2017 relative to 2015 (29) are consistent with acquired immunity blunting the efficiency of transmission, as suggested by Netto et al. (24).

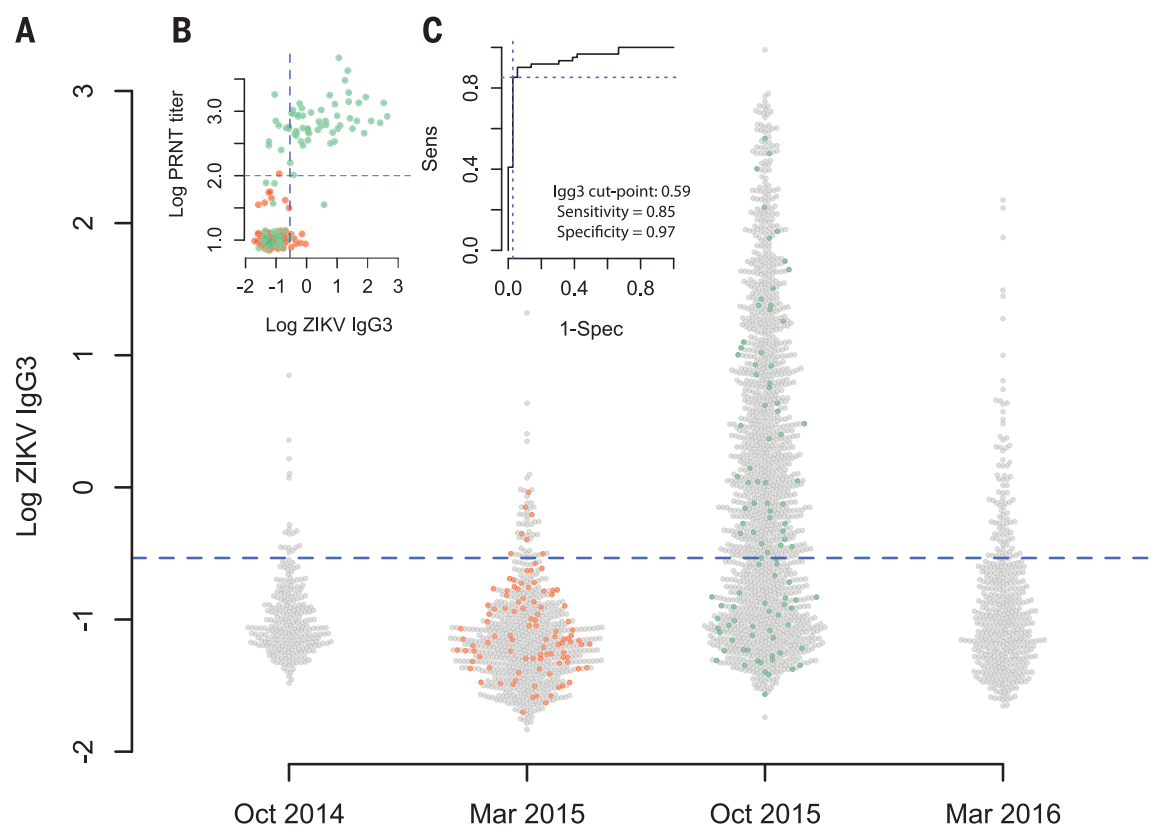

Fig. 2. Results of serological testing conducted before and after the ZIKV outbreak among Pau da Lima cohort participants. (A) Each dot shows the result of an individual participant sample using our ZIKV IgG3 assay (see supplementary materials). (B) ZIKV PRNT was performed on a random subset of samples collected in March 2015 (orange) and October 2015 (green). (C) ROC curve showing the performance of the ZIKV IgG3 assay. Dashed line indicates the cutoff established, using the PRNT results as the gold standard, to achieve a sensitivity of $85 \%$ and a specificity of $97 \%$.

\section{Influence of dengue immunity on ZIKV infection}

We investigated potential associations between DENV antibody titers measured before the ZIKV epidemic and the subsequent risk of ZIKV infection. A total of 642 samples collected in March 2015 were tested for total IgG against DENV NS1 protein, a marker of prior DENV infection; 553 samples (86\%) were positive (30). Age-specific seropositivity patterns were consistent with high levels of endemic DENV transmission in this population (Fig. 3B). Among individuals who had prior immunity to DENV, each doubling in total IgG titers against DENV NS1 was associated with a $9 \%(95 \% \mathrm{CI}, 1$ to $17 \%)$ reduction in the risk of ZIKV infection (table S5). A similar trend was observed in a subset of 96 individuals who were tested for DENV neutralizing antibodies using PRNT (26\% reduction; 95\% CI, -18 to $54 \%)$.

To investigate a possible nonlinear effect of DENV IgG titers on the risk of ZIKV infection, 
we estimated the risk of ZIKV infection among individuals in different tertiles of pre-outbreak IgG titers. We found that individuals in the middle and highest tertiles experienced a $38 \%$ (95\% CI, 8 to $59 \%)$ and $44 \%$ (95\% CI, 14 to $63 \%$ ) reduction in the odds of being ZIKV-positive after the outbreak, respectively, relative to individuals in the lowest tertile with no or low titers of DENV IgG (table S4). We did not find any association between age and risk of ZIKV infection, despite the positive association between DENV IgG levels and age that is characteristic of endemic settings $(31,32)$. This contrasts with a study in Nicaragua that found a positive association between age and ZIKV infection risk (33).

We also explored the association between preoutbreak IgG3 antibodies against DENV NS1 and the risk of subsequent ZIKV infection. This subclass of IgG antibodies has been previously shown to be a marker of recent exposure to DENV infection and to be detectable for as long as 4 to 6 months after infection (30). In contrast to total pre-outbreak IgG titers against DENV NS1, IgG3 levels were positively associated with risk of infection by ZIKV. A factor of 2 increase in IgG3 was associated with a $23 \%$ (95\% CI, 7 to $43 \%)$ increase in the odds of being ZIKV-positive after the outbreak, in models adjusted for age and total IgG NS1 titers. To assess whether the observed results could be due to cross-reactivity between pre-outbreak ZIKV IgG3 and DENV IgG3 responses, we performed sensitivity analyses where we adjusted for ZIKV IgG3 levels measured in March 2015 (table S6). This additional adjustment did not affect the results for the positive association between DENV IgG3 and ZIKV seropositivity. This positive association might reflect an immune profile, in individuals who have experienced a recent DENV infection, that is associated with having a greater risk of a subsequent ZIKV infection. Alternatively, it is also possible that higher levels of IgG3 are a proxy for frequent DENV exposure and thus greater risk of infection by Aedes aegypti-transmitted viruses.

To further investigate the association between prior DENV exposure and ZIKV infection, we fit a generalized additive model (GAM) including DENV NS1 total IgG, DENV NS1 IgG3, and age as covariates (Fig. 4A). Consistent with results from logistic models, these analyses showed a negative linear association between pre-outbreak anti-DENV NS1 total IgG (on the log scale) and subsequent ZIKV infection, and a positive linear association with pre-outbreak anti-DENV NS1 IgG3. Although age was not associated with risk of ZIKV infection when modeled using logistic regression or GAMs, we did find a nonlinear effect of age on the ratio of ZIKV IgG3 levels measured after and before the outbreak (Fig. 4B). The average ratio was 3.8 (95\% CI, 3.0 to 4.6 ) in children aged 5 to 10 years but was 2.4 (95\% CI, 2.1 to 2.8$)$ in individuals older than 20 years of age. This difference remained significant in models adjusted for ZIKV IgG3 levels prior to the outbreak.

We also investigated associations between prior DENV immunity and self-reported Zika symptoms recorded from the cohort. Among
A

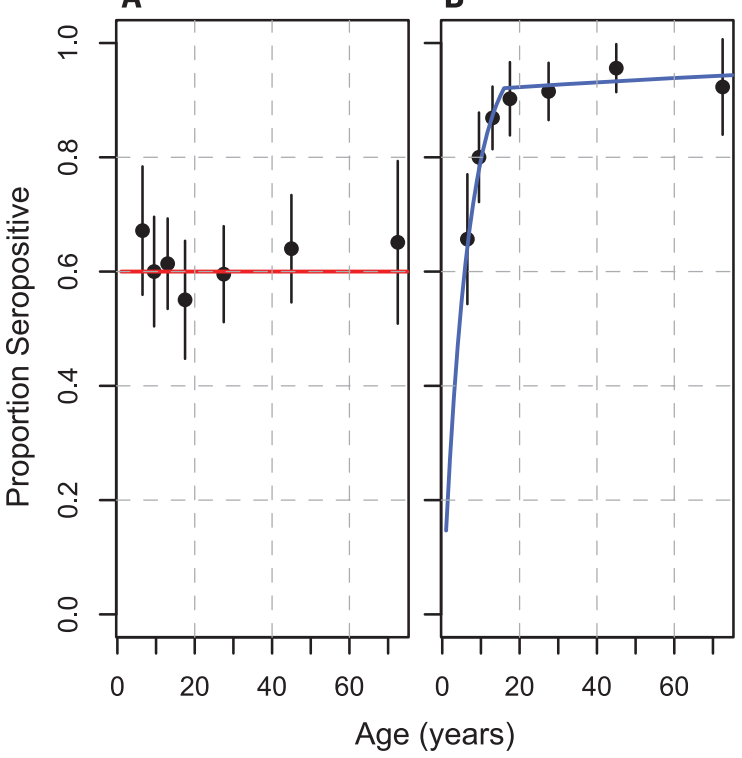

Fig. 3. Age-stratified seropositivity to ZIKV and DENV measured in October 2015 (post-Zika outbreak) and March 2015 (pre-Zika outbreak), respectively. (A) ZIKV seropositivity. The red line shows the mean seropositivity in this population. (B) DENV seropositivity. The blue line shows the fit of the best model estimating the historical DENV force of infection in this population (see supplementary materials). Error bars denote 95\% confidence intervals.
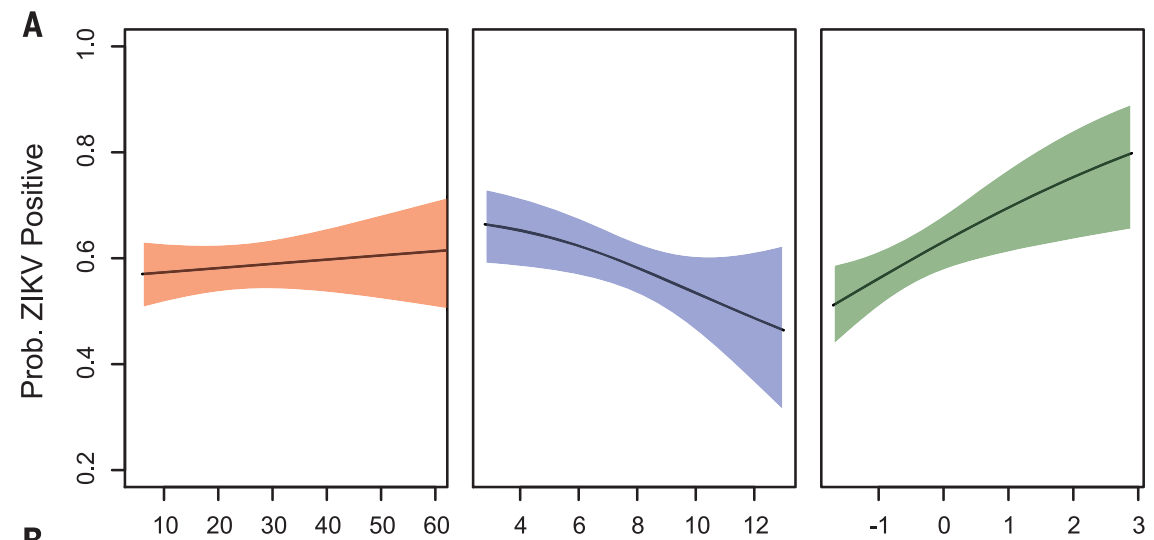

B
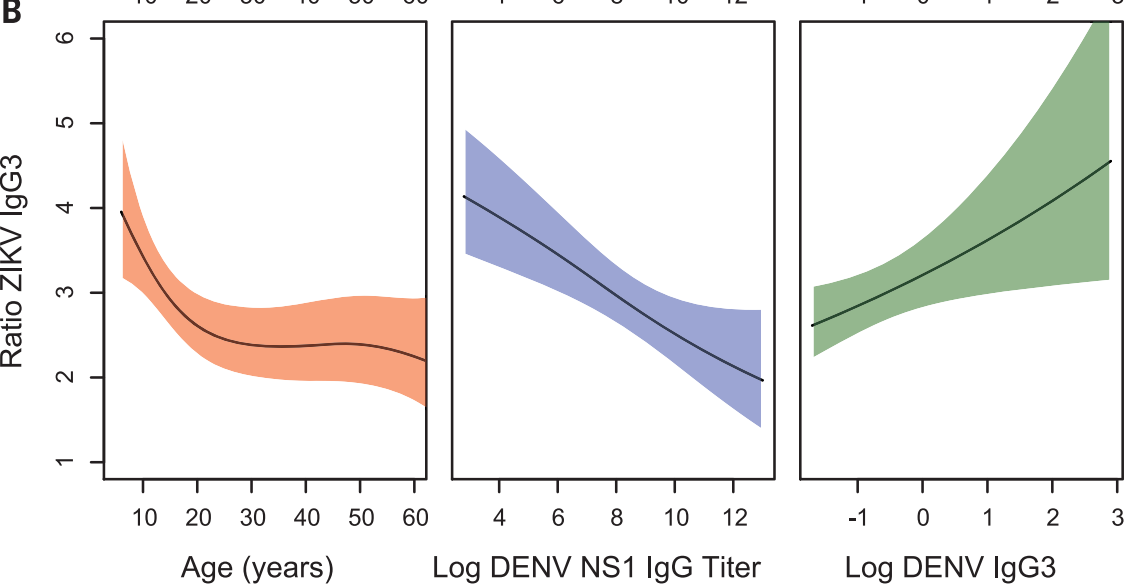

Fig. 4. Results of generalized additive models looking at two metrics of ZIKV infection as a function of age and prior DENV exposure. DENV NS1 lgG titer and DENV NS1 lgG3 levels were measured in March 2015. (A) Proportion ZIKV-positive in October 2015 according to our ZIKV IgG3 assay. (B) Ratio of IgG3 levels in October 2015 versus March 2015. Shaded areas denote 95\% confidence intervals. 
642 cohort individuals, 29 (5\%), 206 (32\%), and $11(2 \%)$ reported experiencing an acute episode of skin rash, fever, or both when interviewed after the Zika epidemic during the October 2015 survey, consistent with at most $36 \%$ of individuals presenting any symptoms. ZIKV seropositivity at the time of interview was associated with reporting rash [odds ratio (OR), 3.17; 95\% CI, 1.19 to 8.43] but not with fever alone (OR, $0.99 ; 95 \% \mathrm{CI}$, 0.71 to 1.40 ). All 11 individuals who reported both rash and fever during this visit were ZIKVseropositive. In addition, among individuals who were infected by ZIKV, the presence of high antiDENV NS1 total IgG titers prior to the outbreak was associated with $47 \%$ (95\% CI, 10 to $69 \%$ ) reduction in the odds of fever, but no reduction in the odds of rash (tables S8 and S9). Preoutbreak anti-DENV NS1 IgG3 levels were not associated with decreased odds of fever or rash.

\section{Conclusions}

We found that our cohort of individuals residing at the epicenter of the Zika epidemic in Brazil was heavily affected in 2015 , with an estimated attack rate of $73 \%$ (95\% CI, 70 to $76 \%$ ). The pattern of cases observed is consistent with the emergence of a highly transmissible and mostly asymptomatic pathogen, followed by reduced transmission once a large proportion of individuals became immune. These findings provide empirical support for the hypothesis that accumulated immunity drove ZIKV to local extinction by reducing the efficiency of transmission. Spatial heterogeneity in ZIKV attack rates shows that focal pockets of susceptible individuals may remain after the apparent end of the epidemic. Although these populations could sustain some ZIKV transmission and may represent an opportunity for vaccine trials, the overall high rates of immunity will present a barrier for future ZIKV incidence in this community.

\section{REFERENCES AND NOTES}

1. J. Lessler, L. H. Chaisson, L. M. Kucirka, O. Bi, K. Grantz, H. Salje, A. C. Carcelen, C. T. Ott, J. S. Sheffield, N. M. Ferguson, D. A. T. Cummings, C. J. E. Metcalf, I. Rodriguez-Barraquer, Science 353, aaf8160 (2016).

2. N. M. Ferguson, Z. M. Cucunubá, I. Dorigatti, G. L. Nedjati-Gilani, C. A. Donnelly, M.-G. Basáñez, P. Nouvellet, J. Lessler, Science 353, 353-354 (2016).

3. W. Dejnirattisai, P. Supasa, W. Wongwiwat, A. Rouvinski, G. Barba-Spaeth, T. Duangchinda, A. Sakuntabhai, V.-M. Cao-Lormeau, P. Malasit, F. A. Rey, J. Mongkolsapaya, G. R. Screaton, Nat. Immunol. 17, 1102-1108 (2016).

4. G. Barba-Spaeth, W. Dejnirattisai, A. Rouvinski, M.-C. Vaney, I. Medits, A. Sharma, E. Simon-Lorière, A. Sakuntabhai V.-M. Cao-Lormeau, A. Haouz, P. England, K. Stiasny, J. Mongkolsapaya, F. X. Heinz, G. R. Screaton, F. A. Rey, Nature 536, 48-53 (2016).

5. S. V. Bardina, P. Bunduc, S. Tripathi, J. Duehr, J. J. Frere, J. A. Brown, R. Nachbagauer, G. A. Foster, D. Krysztof, D. Tortorella, S. L. Stramer, A. García-Sastre, F. Krammer, J. K. Lim, Science 356, 175-180 (2017)

6. P. Pantoja, E. X. Pérez-Guzmán, I. V. Rodríguez, L. J. White O. González, C. Serrano, L. Giavedoni, V. Hodara, L. Cruz, T. Arana, M. I. Martínez, M. A. Hassert, J. D. Brien, A. K. Pinto, A. de Silva, C. A. Sariol, Nat. Commun. 8, 15674 (2017).

7. M. K. McCracken, G. D. Gromowski, H. L. Friberg, X. Lin, P. Abbink, R. De La Barrera, K. H. Eckles, L. S. Garver, M. Boyd, D. Jetton, D. H. Barouch, M. C. Wise, B. S. Lewis, J. R. Currier, K. Modjarrad, M. Milazzo, M. Liu, A. B. Mullins, J. R. Putnak, N. L. Michael, R. G. Jarman, S. J. Thomas, PLOS Pathog. 13, e1006487 (2017)
8. P. M. S. Castanha, E. J. M. Nascimento, C. Braga, M. T. Cordeiro, O. V. de Carvalho, L. R. de Mendonça, E. A. N. Azevedo, R. F. O. França, R. Dhalia, E. T. A. Marques, J. Infect. Dis. 215, 781-785 (2017)

9. M. Montoya, M. Collins, W. Dejnirattisai, L. C. Katzelnick H. Puerta-Guardo, R. Jadi, S. Schildhauer, P. Supasa, S. Vasanawathana, P. Malasit, J. Mongkolsapaya, A. D. de Silva, H. Tissera, A. Balmaseda, G. Screaton, A. M. de Silva, E. Harris, J. Infect. Dis. 218, 536-545 (2018).

10. A. P. Durbin, Trends Immunol. 37, 635-636 (2016)

11. P. Abbink, R. A. Larocca, W. Dejnirattisai, R. Peterson, J. P. Nkolola, E. N. Borducchi, P. Supasa, J. Mongkolsapaya, G. R. Screaton, D. H. Barouch, Nat. Med. 24, 721-723 (2018).

12. C. A. Sariol, M. L. Nogueira, N. Vasilakis, Trends Microbiol. 26 186-190 (2018)

13. D. Musso, D. J. Gubler, Clin. Microbiol. Rev. 29, 487-524 (2016).

14. A. Balmaseda, K. Stettler, R. Medialdea-Carrera, D. Collado X. Jin, J. V. Zambrana, S. Jaconi, E. Cameroni, S. Saborio, F. Rovida, E. Percivalle, S. ljaz, S. Dicks, I. Ushiro-Lumb, L. Barzon, P. Siqueira, D. W. G. Brown, F. Baldanti, R. Tedder, M. Zambon, A. M. B. de Filippis, E. Harris, D. Corti, Proc. Natl. Acad. Sci. U.S.A. 114, 8384-8389 (2017)

15. L. Priyamvada, K. M. Quicke, W. H. Hudson, N. Onlamoon, J. Sewatanon, S. Edupuganti, K. Pattanapanyasat, K. Chokephaibulkit, M. J. Mulligan, P. C. Wilson, R. Ahmed, M. S. Suthar, J. Wrammert, Proc. Natl. Acad. Sci. U.S.A. 113 7852-7857 (2016)

16. M. Kikuti, L. B. Tauro, P. S. S. Moreira, G. S. Campos, I. A. D. Paploski, S. C. Weaver, M. G. Reis, U. Kitron, G. S. Ribeiro, Virol. J. 15, 108 (2018)

17. J. E. Hagan, P. Moraga, F. Costa, N. Capian, G. S. Ribeiro, E. A. Wunder Jr., R. D. M. Felzemburgh, R. B. Reis, N. Nery, F. S. Santana, D. Fraga, B. L. Dos Santos, A. C. Santos, A. Queiroz W. Tassinari, M. S. Carvalho, M. G. Reis, P. J. Diggle, A. I. Ko, PLOS Negl. Trop. Dis. 10, e0004275 (2016)

18. R. B. Reis, G. S. Ribeiro, R. D. M. Felzemburgh, F. S. Santana, S. Mohr, A. X. T. O. Melendez, A. Queiroz, A. C. Santos, R. R. Ravines, W. S. Tassinari, M. S. Carvalho, M. G. Reis, A. I. Ko, PLOS Negl. Trop. Dis. 2, e228 (2008).

19. R. D. M. Felzemburgh, G. S. Ribeiro, F. Costa, R. B. Reis, J. E. Hagan, A. X. T. O. Melendez, D. Fraga, F. S. Santana, S. Mohr, B. L. dos Santos, A. Q. Silva, A. C. Santos, R. R. Ravines, W. S. Tassinari, M. S. Carvalho, M. G. Reis, A. I. Ko, PLOS Negl. Trop. Dis. 8, e2927 (2014).

20. R. Lowe, C. Barcellos, P. Brasil, O. G. Cruz, N. A. Honório, H. Kuper, M. S. Carvalho, Int. J. Environ. Res. Public Health 15 , 96 (2018)

21. M. Kikuti, G. M. Cunha, I. A. D. Paploski, A. M. Kasper, M. M. O. Silva, A. S. Tavares, J. S. Cruz, T. L. Queiroz, M. S. Rodrigues, P. M. Santana, H. C. A. V. Lima, J. Calcagno, D. Takahashi, A. H. O. Gonçalves, J. M. G. Araújo, K. Gauthier, M. A. Diuk-Wasser, U. Kitron, A. I. Ko, M. G. Reis, G. S. Ribeiro, PLOS Negl. Trop. Dis. 9, e0003937 (2015)

22. F. Costa, M. Sarno, R. Khouri, B. de Paula Freitas, I. Siqueira, G. S. Ribeiro, H. C. Ribeiro, G. S. Campos, L. C. Alcântara, M. G. Reis, S. C. Weaver, N. Vasilakis, A. I. Ko, A. R. Almeida, Ann. Intern. Med. 164, 689-691 (2016)

23. C. W. Cardoso, I. A. D. Paploski, M. Kikuti, M. S. Rodrigues, M. M. O. Silva, G. S. Campos, S. I. Sardi, U. Kitron, M. G. Reis, G. S. Ribeiro, Emerg. Infect. Dis. 21, 2274-2276 (2015).

24. E. M. Netto, A. Moreira-Soto, C. Pedroso, C. Höser, S. Funk, A. J. Kucharski, A. Rockstroh, B. M. Kümmerer, G. S. Sampaio, E. Luz, S. N. Vaz, J. P. Dias, F. A. Bastos, R. Cabral, T. Kistemann, S. Ulbert, X. de Lamballerie, T. Jaenisch, O. J. Brady, C. Drosten, M. Sarno, C. Brites, J. F. Drexler, mBio 8, e01390-17 (2017).

25. M. P. Mammen, C. Pimgate, C. J. M. Koenraadt, A. L. Rothman, J. Aldstadt, A. Nisalak, R. G. Jarman, J. W. Jones, A. Srikiatkhachorn C. A. Ypil-Butac, A. Getis, S. Thammapalo, A. C. Morrison, D. H. Libraty, S. Green, T. W. Scott, PLOS Med. 5, e205 (2008).

26. A. J. Kucharski, S. Funk, R. M. Eggo, H.-P. Mallet, W. J. Edmunds, E. J. Nilles, PLOS Negl. Trop. Dis. 10, e0004726 (2016).

27. D. P. Rojas, N. E. Dean, Y. Yang, E. Kenah, J. Quintero, S. Tomasi, E. L. Ramirez, Y. Kelly, C. Castro, G. Carrasquilla, M. E. Halloran, I. M. Longini, Euro Surveill. 21, 30283 (2016)

28. S. Towers, F. Brauer, C. Castillo-Chavez, A. K. I. Falconar, A. Mubayi, C. M. E. Romero-Vivas, Epidemics 17, 50-55 (2016)

29. Salvador Secretary of Health, "Situação Epidemiológica da Dengue, Chikungunya e Zika em Salvador" (number 13, 2018); www.cievs.saude.salvador.ba.gov.br/uploads/1815/.

30. E. J. M. Nascimento et al., J. Virol. Methods 257, 62-68 (2018).

31. N. M. Ferguson, C. A. Donnelly, R. M. Anderson, Philos. Trans. R. Soc. London Ser. B 354, 757-768 (1999).
2. R. M. Anderson, R. M. May, Infectious Diseases of Humans: Dynamics and Control (Oxford Univ. Press, 1992).

33. J. V. Zambrana, Proc. Natl. Acad. Sci. U.S.A. 115, 9294-9299 (2018)

34. I. Rodriguez-Barraquer, F. Costa, Github repository: Code to reproduce main study results, doi:10.5281/ zenodo.2527639.

\section{ACKNOWLEDGMENTS}

We thank the participants of the cohort study and the community leaders from the Pau da Lima Urban Health Council who mobilized support for the investigation; the team members from Oswaldo Cruz Foundation, Brazilian Ministry of Health, who participated in the data collection; the Company for Urban Development of the State of Bahí (CONDER), which provided digital maps of the study site; G. Kuan and the study team at the National Virology Laboratory, the Sócrates Flores Vivas Health Center, the Hospital Infantil Manuel de Jesús Rivera of the Nicaraguan Ministry of Health, and the Sustainable Sciences Institute for their work in collecting and characterizing the samples from the Nicaraguan Pediatric Dengue Cohort Study and the Pediatric Dengue Hospital-based Study in Managua, Nicaragua, that were used in this study; M. Montoya at the University of California, Berkeley, for assistance with sample management; and the World Reference Collection of Emerging Viruses and Arboviruses (WRCEVA) by the Allergan Foundation and the Global Virus Network for providing sera on U.S. travelers. This work could not be accomplished without the joint collaborative effort of the resident associations, community leaders, and residents that constitute the Urban Health Council of Pau da Lima. Funding: Supported by grants from the Yale School of Public Health: Oswaldo Cruz Foundation, Brazilian Ministry of Health; Brazilian National Council for Scientific and Technological Development (400830/2013-2, 440891/ 2016-7, and INCT-Dengue); Coordination for the Improvement of Higher Education Personnel, Brazilian Ministry of Education [440891/2016-7, National Postdoctoral Program (PNPD)]; Research Support Foundation for the State of Bahía (FAPESB PET0026/2013, APP0044/2016, 10206/2015, and PET0022/2016); Research Support Foundation for the State of São Paulo (FAPESP 2013/21719-3 and 2016/15021-1); CuraZika Foundation (curazika. pitt.edu); Wellcome Trust (102330/Z/13/Z); and NIH grants R01 Al121207, R01 TW009504, R01 Al052473, U01 Al088752, R25 TW009338, R01 NS064905, R24 Al120942, U01 Al115577, R01 Al099631, U54 Al065359, P01 Al106695, U19 Al118610, R01 Al114703, and U54 GM088491. S.R.A. was funded in part by the McLaughlin Fellowship Fund. Author contributions: Conceptualization and design of the experiments, A.I.K., I.R.-B., D.A.T.C., E.T.A.M., F.C. M.G.R.; performance of the investigation, F.C., E.J.M.N. N.N., P.M.S.C., G.A.S., J.C., M.C., D.S., J.E.H., H.A., E.A.W D.F.C., S.R.A., S.L.R., N.V.; formal analysis of the data, I.R.-B. F.C., N.N., D.A.T.C., A.I.K.; contribution of resources, S.C.W., G.S.R., A.B., E.H., M.L.N., M.G.R., E.T.A.M., D.A.T.C., A.I.K.; writing, review, and editing of the paper: I.R.-B., F.C., E.J.M.N., E.T.A.M., D.A.T.C., A.I.K. Competing interests: E.J.M.N., E.T.A.M., and A.I.K. are authors on a patent application entitled "Methods and Composition for the Detection of Flavivirus Infections" related to this work (U.S. Provisional Patent Application Serial No. 62/503.201 and U.S. Provisional Patent Application Serial No. 62/608.927). AlK received honoraria from Sanofi-Pasteur for his participation in a conference on Zika vaccines. The authors declare that they have no other competing interests. Data and materials availability: Material transfer agreements exist between Yale University and Fundação Oswaldo Cruz that relate to access to human materials. All relevant data are within the paper and its supporting files. Code to reproduce study findings is available at https://doi.org/10 5281/zenodo.2528486 (34).

\section{SUPPLEMENTARY MATERIALS}

www.sciencemag.org/content/363/6427/607/suppl/DC1 Materials and Methods

Figs. S1 to S3

Tables S1 to $\mathrm{Sg}$

References (35-45)

8 October 2018; accepted 15 January 2019

10.1126/science.aav6618 


\section{Science}

\section{Impact of preexisting dengue immunity on Zika virus emergence in a dengue endemic region}

Isabel Rodriguez-Barraquer, Federico Costa, Eduardo J. M. Nascimento, Nivison Nery Júnior, Priscila M. S. Castanha, Gielson Almeida Sacramento, Jaqueline Cruz, Mayara Carvalho, Daiana De Olivera, José E. Hagan, Haritha Adhikarla, Elsio A.

Wunder Jr., Danilo F. Coêlho, Sasha R. Azar, Shannan L. Rossi, Nikos Vasilakis, Scott C. Weaver, Guilherme S. Ribeiro,

Angel Balmaseda, Eva Harris, Maurício L. Nogueira, Mitermayer G. Reis, Ernesto T. A. Marques, Derek A. T. Cummings and Albert I. Ko

Science 363 (6427), 607-610.

DOI: $10.1126 /$ science.aav6618

\section{Zika dynamics in South America}

The infection dynamics of Zika virus (ZIKV) are difficult to characterize. Many ZIKV infections are asymptomatic, and the clinical presentation of ZIKV is nonspecific. Rodriguez-Barraquer et al. took advantage of a long-term health study under way in Salvador, Brazil, the epicenter of the recent outbreak in the Americas. They used multiple serological assays, from before and after the emergence of ZIKV in October 2015, to distinguish ZIKV immune responses from those against Dengue virus (DENV). About $73 \%$ of the population was attacked by ZIKV. The presence of preexisting antibodies to DENV was associated with less risk of ZIKV infection and fewer symptoms. Science, this issue p. 607

ARTICLE TOOLS

SUPPLEMENTARY MATERIALS

RELATED CONTENT

REFERENCES

PERMISSIONS http://science.sciencemag.org/content/363/6427/607

http://science.sciencemag.org/content/suppl/2019/02/06/363.6427.607.DC1

http://stm.sciencemag.org/content/scitransmed/9/388/eaag0538.full http://stm.sciencemag.org/content/scitransmed/10/444/eaar2749.full http://stm.sciencemag.org/content/scitransmed/8/369/369ra179.full http://stm.sciencemag.org/content/scitransmed/10/435/eaa06975.full

This article cites 39 articles, 9 of which you can access for free http://science.sciencemag.org/content/363/6427/607\#BIBL

http://www.sciencemag.org/help/reprints-and-permissions

Use of this article is subject to the Terms of Service

Science (print ISSN 0036-8075; online ISSN 1095-9203) is published by the American Association for the Advancement of Science, 1200 New York Avenue NW, Washington, DC 20005. The title Science is a registered trademark of AAAS.

Copyright (C 2019 The Authors, some rights reserved; exclusive licensee American Association for the Advancement of Science. No claim to original U.S. Government Works 